\title{
Analysis of $\beta$-Amyloid Peptide -Binding Proteins in Microglial Cells
}

\author{
Sachiko Ito, Naomi Nishio and Ken-Ichi Isobe*
}

Department of Immunology, Nagoya University Graduate School of Medicine, 65 Turumai-cho, Showa-ku, Nagoya, Aichi, 466-8520, Japan

\begin{abstract}
Alzheimer's disease is the most common form of dementia in the elderly. Although $\beta$-amyloid peptide (A $\beta$ ) has been considered major cause of Alzheimer pathology, molecular and cellular mechanisms of the disease development of Alzheimer have not been clarified yet. Presently A $\beta$ has been considered to induce neural cell death by direct penetration of aggregated form or indirect cell death by inflammatory responses induced by $A \beta$-activated microglia. In order to understand $A \beta$ induced microglial activation, we searched the proteins which bind to $A \beta$ in activated microglial cell line. We stimulated Ra2 microglial cell line with $A \beta$. Activated Ra2 cells were immunoprecipitated with anti- $A \beta$ and run the gel. Membrane was silver stained and bands were cut and digested with enzyme. They were analyzed by LC/MS/MS. We found that several proteins including myosin 9 and actin bound to $A \beta$. By the addition of $A \beta$, actin binding was enhanced and other proteins including IQGAP1, Plectin strongly bound to $A \beta$. These results indicate that $A \beta$ binds to the proteins belonging to cellular cytoskeletal system.
\end{abstract}

Keywords: Alzheimer, Abeta, LC/MS/MS, microglia, IQGAP1, actin.

\section{INTRODUCTION}

Alzheimer's disease is the most common form of dementia. More than 35 million people have Alzheimer's disease worldwide. The principal risk factor for Alzheimer's disease is age [1]. Cerebral plaques laden with $\beta$-amyloid peptide $(A \beta)$ have been considered major cause of Alzheimer pathology [2]. $A \beta$ outside the cell spontaneously selfaggregates. $A \beta$ can also grow into fibrils, which arrange themselves into $\beta$-pleated sheets to form the insoluble fibers of advanced amyloid plaques. $A \beta$ damages neurons by two ways. One is direct toxic effect of $A \beta$ to neurons. Another is indirect damages to neurons by microglial activation.

$\mathrm{A} \beta$ binds to CD36 and other scavenger receptors on microglia, which may induce microglial activation and produce inflammatory cytokines and chemokines, reactive oxygen species (ROS) and reactive nitrogen species (RNS) and other neurotoxins [3,4]. CD36 has established roles in the endocytic uptake of altered self components, including oxidized phospholipids, apoptotic cells and amyloid proteins [5] Recently it has been shown that CD36-TLR4-TLR6 activation as a common molecular mechanism by which atherogenic lipids and $A \beta$ stimulate sterile inflammation [6]. We have shown that $A \beta$ 25-35 induces Akt and I $\kappa \mathrm{B} \alpha$ phosphorylation within $15 \mathrm{~min}$ after addition to culture medium [7]. Further A $\beta$ 1-42 induces Akt and PI3/Akt phosphorylation within $5 \mathrm{~min}[8]$. These results indicate that monomer or soluble oligomer $A \beta$ but not aggregated $A \beta$ bind to cell membrane and induces immflamatory responses. In these cases $A \beta$ may stay in cell membrane or enter cells by endocytosis. It has been postulated that in neuronal cells $\mathrm{A} \beta$ works inside cells and may dampen excitatory

*Address correspondence to this author at the Department of Immunology, Nagoya University Graduate School of Medicine, 65 Turumai-cho, Showaku, Nagoya, Aichi, 466-8520, Japan; Tel: +81-52-744-2135; Fax: +81-52744-2972; E-mail: kisobe@med.nagoya-u.ac.jp transmission and prevent neuronal hyperactivity [9]. In order to understand the $A \beta$-induced microglial activation, we tried to find out $\mathrm{A} \beta$ binding proteins inside microglia by LC/MS/MS methods.

\section{MATERIALS AND METHODS}

\section{Materials}

Synthetic human A $\beta 1-42$ was obtained from Peptide Institute Inc (Osaka, Japan). A $\beta 1-42$ was dissolved in $0.1 \%$ $\mathrm{NH}_{3}$ according to the manufacturer's instructions. Mouse monoclonal anti-A $\beta$ antibody (Millipore, MAB1561) was used for Immunoprecipitation. This monoclonal anitibody (IgG2b) binds to human and mouse $A \beta$. The epitope lies between amino acids 18-22 of $\mathrm{A} \beta$.

\section{Cell Culture}

Microglial cell line Ra2 was cultured in MGI medium [Eagle's MEM supplemented with $0.2 \%$ glucose, $5 \mu \mathrm{g} / \mathrm{ml}$ bovine Insulin (Sigma-Aldrich), and 10\% fetal bovine serum (FBS, Invitrogen)] and $0.8 \mathrm{ng} / \mathrm{ml} \mathrm{mrGM}-\mathrm{CSF}$ (Pharmingen) [10].

\section{Immunoprecipitation}

The cells were lysed with RIPA buffer $(25 \mathrm{mM}$ Tris- $\mathrm{HCl}$, pH8.0, $150 \mathrm{mM} \mathrm{NaCl}, 10 \%$ glycerol, $2 \mathrm{mM}$ EDTA, $5 \mathrm{mM}$ $\mathrm{MgCl}_{2}, 0.3 \% \mathrm{NP} 401 \mathrm{mM}$ PMSF). $5 \mathrm{mg}$ of affinity-purified monoclonal antibodies was covalently coupled to $50 \mathrm{~mL}$ Protein $\mathrm{G}$ beads (GE healthcare) by using $20 \mathrm{mM}$ DMP. Cell lysates were pre-incubated with protein $\mathrm{G}$ beads for 2 hour at $4{ }^{\circ} \mathrm{C}$ and then centrifuged. The supernatant was incubated with antibody-cross-linked protein $\mathrm{G}$ beads overnight at 4 ${ }^{\circ} \mathrm{C}$. The beads were washed five times with RIPA buffer and then suspended in $0.1 \mathrm{M}$ glycine $(\mathrm{pH} 1.7)$ at $4{ }^{\circ} \mathrm{C}$ for $1 \mathrm{hr}$. The eluted sample was neutralized by the addition of $1 \mathrm{M}$ Tris- $\mathrm{HCl}(\mathrm{pH} 9.0)$. The eluted proteins were mixed with sample buffer (62.5 mM Tris-HCl, pH 6.8, 2\% SDS, 10\% 
glycerol, 5\% 2-mercaptoethanol, and 5\% bromophenol blue). A fraction of the eluate was monitored by SDS-PAGE and silver stained.

\section{Identification of Proteins}

For LC-MS/MS ion search analysis, protein spots were excised from the gel. The gel pieces were destained and dried by vacuum centrifugation. For carbamidomethyl modification, the dried gel pieces were rehydrated in 100 $\mathrm{mM}$ ammonium bicarbonate containing $10 \mathrm{mM}$ DTT. After removal of the solution, the gel pieces were alkylated and then rehydrated in a trypsin digest solution (Trypsin Gold, Mass Spectrometry Grade (Promega Co., Madison, WI)). LC-MS/MS ion search analysis was performed using an LCQ Advantage nanospray ionization iontrap mass spectrometer (Thermo Fisher Scientific, Inc., Waltham, MA) combined with a MAGIC2002TM HPLC System (Michrom BioResources, Inc., Auburn, CA) that was equipped with a MonoCapR column of $0.1 \mathrm{~mm}$ diameter and $50 \mathrm{~mm}$ in length (AMR Inc., Tokyo Japan). The MS/MS spectrum data collected repeatedly were submitted to the program Mascot (Matrix Science Inc., Boston, MA).

\section{RESULTS}

\section{Several Proteins Bind to Aß 1-42 in Microglial Cells}

We used affinity separation techniques using anti- $\mathrm{A} \beta$ bound to beads to fractionate extracts from Ra2 cells (Fig. 1). In this procedure, we added $A \beta 1-42$ in lysis of Ra 2 cells. The bound proteins were run in SDS PAGE and stained with silver. Several bands were detected. Without A $\beta$ 1-42 we could detect several bands (Fig. 2 midle lane). By the addition of $A \beta$ 1-42, many new bands appeared (Fig. 2, right). Among them we took four bands. Band No1 and No3 appeared only in the lane of $A \beta 1-42$ addition. Band No2 and No4 appeared both in the lane with $A \beta$ 1-42 and without $A \beta$ $1-42$. However, No4 band is stronger in the lane with $A \beta 1$ 42 than in the lane without A $\beta$ 1-42 (Fig. 2).

\section{IQGAP1 and Plectin Bind to A $\beta$ 1-42 in Microglial Cells}

Gel bands were cut out and purified. Extracted trypsindigested proteins of the samples were analyzed by LCMS/MS Band. No2 and No4, which were found both in Ra2 microglia with or without $A \beta 1-42$, were myosin 9 (No2) (Fig. 3A) and actin (No4) (Fig. 3B).

No3, which was strongly expressed in Ra2 microglia with A $\beta$ 1-42, was IQGAP1 (Fig. 3C). No1, which expressed in Ra2 microglia with $A \beta$ 1-42, was Plectin (Fig. 3D).

\section{DISCUSSION}

\section{A $\beta$ binds to Several Proteins in Microglial Cells}

By immunoprecipitation using anti-A $\beta$ antibody, we found four strong bands without addition of $A \beta$ 1-42. Among them No2 was analyzed by LC/MS/MS and found to be myosin 9. This band was not enhanced by the addition of $\mathrm{A} \beta$. However, No4 protein was greatly enhanced by the addition of $A \beta$. No4 protein was found to be actin. These results indicate that the addition of $A \beta$ enhances actin expression. We speculate that $\mathrm{A} \beta$ 1-42 induces cellular migration, which induces F-actin in activated cells. Addition of A $\beta$ 1-42 induces many new proteins in microglial cells. By the addition of $A \beta 1-42$, we detected several new bands.
Among them we determined most strong band (No3). By the analysis of LC/MS/MS, we found that it was IQGAP1.

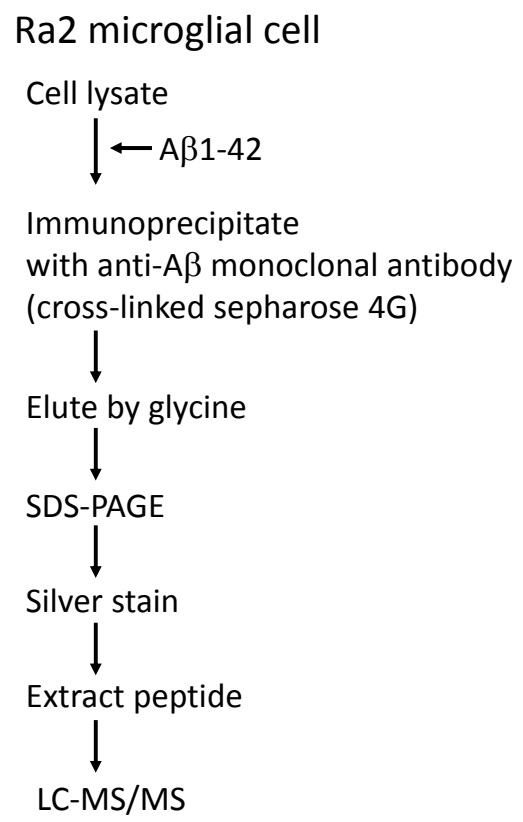

Fig. (1). Flow diagram for proteomic isolation of $A \beta$ binding proteins. Ra2 cells were lysed in RIPA buffer, which were immunoprecipitated with anti-Ab and run the gel. Stained gel bands were cut and digested for LC/MS/MS.

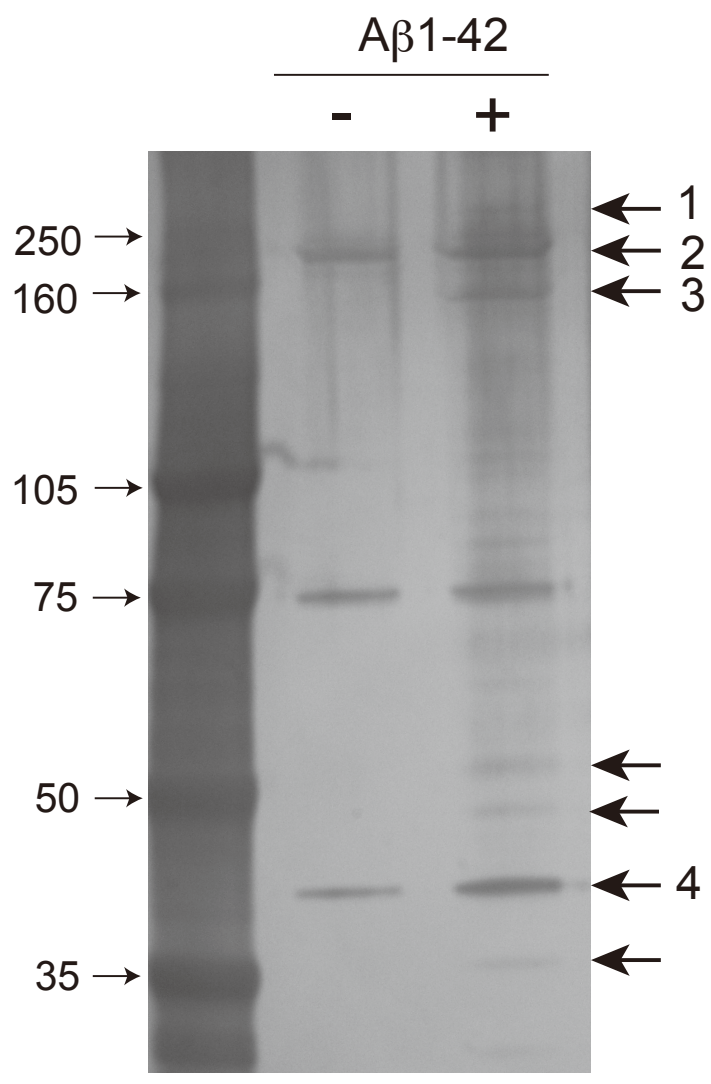

Fig. (2). Several proteins bind to $A \beta$ by immunoprecipitation. Ra2 extracts were treated with anti $A \beta$ antibody cross-linked protein $G$ beads. The bound proteins were stripped from the beads and run on SDS-PAGE gels and stained with silver. 


\section{Myosin-9 sequence}

MAQQAADKYL YVDKNFINNP LAQADWAAKK LVWVPSSKNG FEPASLKEEV GEEAIVELVE NGKKVKVNKD DIQKMNPPKF SKVEDMAELT CLNEASVLHN LKERYYSGLI YTYSGLFCVV INPYKNLPIY SEEIVEMYKG KKRHEMPPHI YAITDTAYRS MMQDREDQSI LCTGESGAGK TENTKKVIQY LAHVASSHKS KKDQGELERQ LLQANPILEA FGNAKTVKND NSSRFGKFIR INFDVNGYIV GANIETYLLE KSRAIRQAKE ERTFHIFYYL LSGAGEHLKT DLLLEPYNKY RFLSNGHVTI PGQQDKDMFQ ETMEAMRIMG IPEDEQMGLL RVISGVLQLG NIAFKKERNT DQASMPDNTA AQKVSHLLGI NVTDFTRGIL TPRIKVGRDY VQKAQTKEQA DFAIEALAKA TYERMFRWLV LRINKALDKT KRQGASFIGI LDIAGFEIFD LNSFEQLCIN YTNEKLQQLF NHTMFILEQE EYQREGIEWN FIDFGLDLQP CIDLIEKPAG PPGILALLDE ECWFPKATDK SFVEKVVQEQ GTHPKFQKPK QLKDKADFCI IHYAGKVDYK ADEWLMKNMD PLNDNIATLL HQSSDKFVSE LWKDVDRIIG LDQVAGMSET ALPGAFKTRK GMFRTVGQLY KEQLAKLMAT LRNTNPNFVR CIIPNHEKKA GKLDPHLVLD QLRCNGVLEG IRICRQGFPN RVVFQEFRQR YEILTPNSIP KGFMDGKQAC VLMIKALELD SNLYRIGQSK VFFRAGVLAH LEEERDLKIT DVIIGFQACC RGYLARKAFA KRQQQLTAMK VLQRNCAAYL RLRNWQWWRL FTKVKPLLNS IRHEDELLAK EAELTKVREK HLAAENRLTE METMQSQLMA EKLQLQEQLQ AETELCAEAE ELRARLTAKK QELEEICHDL EARVEEEEER CQYLQAEKKK MQQNIQELEE QLEEEESARQ KLQLEKVTTE AKLKKLEEDQ IIMEDQNCKL AKEKKLLEDR VAEFTTNLME EEEKSKSLAK LKNKHEAMIT DLEERLRREE KQRQELEKTR RKLEGDSTDL SDQIAELQAQ IAELKMQLAK KEEELQAALA RVEEEAAQKN MALKKIRELE TQISELQEDL ESERASRNKA EKQKRDLGEE LEALKTELED TLDSTAAQQE LRSKREQEVS ILKKTLEDEA KTHEAQIQEM RQKHSQAVEE LADQLEQTKR VKATLEKAKQ TLENERGELA NEVKALLQGK GDSEHKRKKV EAQLQELQVK FSEGERVRTE LADKVTKLQV ELDSVTGLLS QSDSKSSKLT KDFSALESQL QDTQELLQEE NRQKLSLSTK LKQMEDEKNS FREQLEEFEE AKRNLEKQIA TLHAQVTDMK KKMEDGVGCL ETAEEAKRRL QKDLEGLSQR LEEKVAAYDK LEKTKTRLQQ ELDDLLVDLD HQRQSVSNLE KKQKKFDQLL AEEKTISAKY AEERDRAEAE AREKETKALS LARALEEAME QKAELERLNK QFRTEMEDLM SSKDDVGKSV HELEKSKRAL EQQVEEMKTQ LEELEDELQA

TEDAKLRLEV NLQAMKAQFE RDLQGRDEQS EEKKKQLVRQ VREMEAELED ERKQRSMAMA ARKKLEMDLK DLEAHIDTAN KNREEAIKQL RKLQAQMKDC MRELDDTRAS REEILAQAKE NEKKLKSMEA EMIQLQEELA AAERAKRQAQ QERDELADEI ANSSGKGALA LEEKRRLEAR IAQLEEELEE EQGNTELIND RLKKANLQID QINTDLNLER SHAQKNENAR QQLERQNKEL KAKLQEMESA VKSKYKASIA ALEAKIAQLE EQLDNETKER QAASKQVRRT EKKLKDVLLQ VEDERRNAEQ FKDQADKAST RLKQLKRQLE EAEEEAQRAN ASRRKLQREL EDATETADAM NREVSSLKNK LRRGDLPFVV TRRIVRKGTG DCSDEEVDGK ADGADAKAAE

Actin sequence

(B)

MEEEIAALVI DNGSGMCKAG FAGDDAPRAV FPSIVGRPRH QGVMVGMGQK DSYVGDEAQS KRGILTLKYP IEHGIVTNWD DMEKIWHHTF YNELRVAPEE HPVLLTEAPL NPKANREKMT QIMFETFNTP AMYVAIQAVL SLYASGRTTG IVMDSGDGVT HTVPIYEGYA LPHAILRLDL AGRDLTDYLM KILTERGYSF TTTAEREIVR DIKEKLCYVA LDFEQEMATA ASSSSLEKSY ELPDGQVITI GNERFRCPEA LFQPSFLGME SCGIHETTFN SIMKCDVDIR KDLYANTVLS GGTTMYPGIA DRMQKEITAL APSTMKIKII APPERKYSVW IGGSILASLS TFQQMWISKQ EYDESGPSIV HRKCF 
(Fig. 3) contd.....

\section{IQGAP1 sequence}

(C)

MSAAEEVDGL GVVRPHYGSV LDNERLTAEE MDERRRQNVA YEYLCHLEEA KRWMEACLGE DLPPTTELEE GLRNGVYLAK LGNFFSPKVV SLKKIYDREQ TRYKATGLHF RHTDNVIQWL NAMDEIGLPK IFYPETTDIY DRKNMPRCIY CIHALSLYLF KLGLAPQIQD LYGKVDFTEE EINNMKIELE KYGIQMPAFS KIGGILANEL SVDEAALHAA VIAINEAIDR RVAADTFTAL KNPNAMLVNL EEGLAPTYQD VLYQAKQDKM TNAKNRTENS DRERDVYEEL LTQAEIQGNV NKVNTSSALA NISLALEQGC AVTLLKALQS LALGLRGLQT QNSDWYMKQL QSDLQQKRQS GQTDPLQKEE VQAGVDAANS AAQQYQRRLA AVAAINAAIQ KGIAEKTVLE LMNPEAQLPQ VYPFAADLYQ KELATLQQQS PEHSLTHPEL TVAVEMLSSV ALINRALESG DMTTVWKQLS SSVTGLTNIE EENCQRYLDE LMKLKAQAHA ENNAFITWND IQACVDHVNL VVHEEHERIL AIGLINEALD EGDAQKTLQA LQIPAAKLEG VLAEVAQHYQ DTLIRAKREK AQETQDESAV LWLDEIQGGI WQSNKDTQEA QRFALGISAI NEAVDSGDVG RTLSALRSPD VGLYGVIPEC GETYQSDLAE AKKKRLAAGD NNSKWVKHWV KGGYHYYHNL ETQAGGWAEP PDFVQNSVQL SREEIQSSIS GVTAAYNREQ LWLANEGLIT KLQACCRGYL VRQEFRSRMN FLKKQIPAIT CIQSQWRGYK QKKAYQDRLA YLHSHKDEVV KIQSLARMHQ ARKRYRDRLQ YFRDHINDII KIQAFIRANK ARDDYKTLIN AEDPPMIVVR KFVHLLDQSD QDFQEELDLM KMREEVITLI RSNQQLENDL NLMDIKIGLL VKNKITLQDV VSHSKKLTKK NKEQLSDMMM INKQKGGLKA LSKEKREKLE AYQHLFYLLQ TNPTYLAKLI FQMPQNKSTK FMDSVIFTLY NYASNQREEY LLLRLFQTAL QEEIKSKVDQ IQEIVTGNPT VIKMVVSFNR GARGQNALRQ ILAPVVKEIM DDKSLNIKTD PVDIYKSWVN QMESQTGEAS KLPYDVTPEQ ALSHEEVKTR LDNSIRNMRA VTDKFLSAIV SSVDKIPYGM RFIAKVLKDS LHEKFPDAGE DELLKIIGNL LYYRYMNPAI VAPDAFDIID LSAGGQLTTD QRRNLGSIAK MLQHAASNKM FLGDNAHLSI INEYLSQSYQ KFRRFFQLAC DVPELQDKFN VDEYSDLVTL TKPVIYISIG EIINTHTLLL DHODAIAPEH NDPIHELLDD LGEVPTIESL IGESCGNSND PNKEALAKTE VSLTLTNKFD VPGDENAEMD ARTILLNTKR LIVDVIRFQP GETLTEILET PATNEQEAEH QRAMQRRAIR DAKTPDKMKK SKPMKEDNNL SLQEKKEKIQ TGLKKLTELG TVDPKNRYQE LINDIAKDIR NQRRYRQRRK AELVKLQQTY SALNSKATFY GEQVDYYKSY IKTCLDNLAS KGKVSKKPRE MKGKKSKKIS LKYTAARLHE KGVLLEIEDL QANQFKNVIF EIGPTEEVGD FEVKAKFMGV QMETFMLHYQ DLLQLQYEGV AVMKLFDRAK VNVNLLIFLL NKKFYGK

\section{Plectin sequence}

(D)

MVAGMLMPLD RLRAIYEVLF REGVMVAKKD RRPRSLHPHV PGVTNLQVMR AMASLKARGL VRETFAWCHF YWYLTNEGID HLRQYLHLPP EIVPASLQRV RRPVAMVIPA RRRSPHVQTM QGPLGCPPKR GPLPAEDPAR EERQVYRRKE REEGAPETPV VSATTVGTLA RPGPEPAPAT DERDRVQKKT FTKWVNKHLI KHWRAEAQRH ISDLYEDLRD GHNLISLLEV LSGDSLPREK GRMRFHKLQN VQIALDYLRH RQVKLVNIRN DDIADGNPKL TLGLIWTIIL HFQISDIQVS GQSEDMTAKE KLLLWSQRMV EGYQGLRCDN FTTSWRDGRL FNAIIHRHKP MLIDMNKVYR QTNLENLDQA FSVAERDLGV TRLLDPEDVD VPQPDEKSII TYVSSLYDAM PRVPGAQDGV RANELQLRWQ EYRELVLLLL QWIRHHTAAF EERKFPSSFE EIEILWCQFL KFKETELPAK EADKNRSKVI YQSLEGAVQA GQLKIPPGYH PLDVEKEWGK LHVAILEREK QLRSEFERLE CLQRIVSKLQ MEAGLCEEQL NQADALLQSD IRLLASGKVA QRAGEVERDL DKADGMIRLL FNDVQTLKDG RHPQGEQMYR RVYRLHERLV AIRTEYNLRL KAGVGAPVTQ VTLQSTQRRP ELEDSTLRYL QDLLAWVEEN QRRIDSAEWG VDLPSVEAQL GSHRGMHQSI EEFRAKIERA RNDESQLSPA TRGAYRDCLG RLDLQYAKLL NSSKARLRSL ESLHGFVAAA TKELMWLNEK EEEEVGFDWS DRNTNMAAKK ESYSALMREL EMKEKKIKEI QNTGDRLLRE DHPARPTVES FQAALQTQWS WMLQLCCCIE AHLKENTAYF QFFSDVREAE EQLQKLQETL RRKYSCDRTI TVTRLEDLLQ DAQDEKEQLN EYKGHLSGLA KRAKAIVQLK PRNPAHPVRG HVPLIAVCDY KQVEVTVHKG DQCQLVGPAQ PSHWKVLSGS SSEAAVPSVC FLVPPPNQEA QEAVARLEAQ HQALVTLWHQ LHVDMKSLLA WQSLSRDIQL IRSWSLVTFR TLKPEEQRQA LRNLELHYQA FLRDSQDAGG FGPEDRLVAE REYGSCSRHY QQLLQSLEQG EQEESRCQRC ISELKDIRLQ LEACETRTVH RLRLPLDKDP ARECAQRIAE QQKAQAEVEG LGKGVARLSA EAEKVLALPE PSPAAPTLRS ELELTLGKLE QVRSLSAIYL EKLKTISLVI RSTQGAEEVL KTHEEQLKEA QAVPATLQEL EATKASLKKL RAQAEAQQPV FNTLRDELRG AQEVGERLQQ RHGERDVEVE RWRERVTQLL ERWQAVLAQT DVRQRELEQL GRQLRYYRES ADPLSAWLQD AKRRQEQIQA VPIANCQAAR EQLRQEKALL EEIERHGEKV EECQKFAKQY INAIKDYELQ LITYKAQLEP VASPAKKPKV QSGSESVIQE YVDLRTRYSE LTTLTSQYIK FISETLRRME EEERLAEQQR AEERERLAEV EAALEKQRQL AEAHAQAKAQ AELEAQELQR RMQEEVARRE 
Fig. 3) contd.....

(D)

EAAVDAQQQK RSIQEELQHL RQSSEAEIQA KAQQVEAAER SRMRIEEEIR VVRLQLETTE RQRGGAEGEL QALRARAEEA EAQKRQAQEE AERLRRQVQD ESQRKRQAFA ELALRVKAEA EAAREKQRAL QALDELRLQA EEAERRLRQA EAERARQVQV ALETAQRSAE VELQSKRASF AEKTAQLERT LQEEHVTVAQ LREEAERRAQ QQAEAERARE EAERELERWQ LKANEALRLR LQAEEVAQQK SLAQADAEKQ KEEAEREARR RGKAEEQAVR QRELAEQELE KQRQLAEGTA QQRLAAEQEL IRLRAETEQG EQQRQLLEEE LARLQHEATA ATQKRQELEA ELAKVRAEME VLLASKARAE EESRSTSEKS KQRLEAEAGR FRELAEEAAR LRALAEEAKR QRQLAEEDAA RQRAEAERVL TEKLAAISEA TRLKTEAEIA LKEKEAENER LRRLAEDEAF QRRRLEEQAA LHKADIEERL AQLRKASESE LERQKGLVED TLRQRRQVEE EIMALKVSFE KAAAGKAELE LELGRIRSNA EDTMRSKEQA ELEAARQRQL AAEEEQRRRE AEERVQRSLA AEEEAARQRK VALEEVERLK AKVEEARRLR ERAEQESARQ LQLAQEAAQK RLQAEEKAHA FVVQQREEEL QQTLQQEQNM LDRLRSEAEA ARRAAEEAEE AREQAEREAA QSRKQVEEAE RLKQSAFEQA QAQAQAQAAA EKLRKEAEQE AARRAQAEQA ALKQKQAADA EMEKHKKFAE QTLRQKAQVE QELTTLRLQL EETDHQKSIL DEELQRLKAE VTEAARQRSQ VEEELFSVRV QMEELGKLKA RIEAENRALI LRDKDNTQRF LEEEAEKMKQ VAEEAARLSV AAQEAARLRQ LAEEDLAQQR ALAEKMLKEK MQAVQEATRL KAEAELLQQQ KELAQEQARR LQEDKEQMAQ QLVEETQGFQ RTLEAERQRQ LEMSAEAERL KLRMVEMSRA QARAEEDAQR FRKQAEEIGE KLHRTELATQ EKVTLVQTLE IQRQQSDHDA ERLREAIAEL EREKEKLKQE AKLLQLKSEE MQTVQQEQIL QETQALQKSF LSEKDSLLQR ERFIEQEKAK LEQLFQDEVA KAKQLREEQQ RQQQQMEQEK QELMASMEEA RRRQREAEEG VRRKQEELQH LEQQRQQQEK LLAEENQRLR ERLQRLEEEH RAALAHSEIA TTQAASTKAL PNGRDAPDGP SVEAEPEYTF EGLRQKVPAQ QLQEAGILSQ EELQRLAQGH TTVAELTQRE DVYRYLKGRS SIAGLLLKPT NEKLSVYTAL QRQLLSPGTA LILLEAQAAS GFLLDPVRNR RLTVNEAVKE GVVGPELHHK LLSAERAVTG YKDPYTGEQI SLFQAMKKDL IVRDHGVRLL EAQIATGGII DPVHSHRVPV DVAYKRGYFD EEMNRILSDP SDDTKGFFDP NTHENLTYLQ LLERCVEDPE TGLRLLPLTD KAAKGGELVY TDTEARDVFE KATVSAPFGK FQGRTVTIWE IINSEYFTAE QRRDLLQQFR TGHITVEKII KIVITVVEEH ERKGQLCFEG LRALVPAAEL LDSGVISHEL YQQLQRGERS VREVAEADSV RQALRGTNVI AGVWLEEAGQ KLSIYEALKK DLLQPEVAVA LLEAQAGTGH IIDPATSARL TVDEAVRAGL VGPELHEKLL SAEKAVTGYR DPYSGQSVSL FQALKKGLIP REQGLRLLDA QLSTGGIVDP SKSHRVPLDV AYARGYLDKE TNRALTSPRD DARVYHDPST QEPVTYSQLQ QRCRSDQLTG LSLLPLSEKA VRARQEEVYS ELQARETLEQ AKVEVPVGSF KGRAMTVWEL ISSEYFTEEQ RQELLRQFRT GKVTVEKVIK IVITIVEEVE TRRQERLSFS GLRAPVPASE LLDAKILSRA QFDQLKDGKT SVKELSEVGS VRTLLQGSGC LAGIYLEDSK EKVTIYEAMR RGLLRPSTAT LLLEAQAATG FLVDPVRNQR LYVHEAVKAG VVGPELHEKL LSAEKAVTGY KDPYSGNTIS LFQAMKKGLV LRDHAIRLLE AQVATGGIID PVHSHRLPVD VAYQRGYFDE EMNRVLADPS DDTKGFFDPN THENLTYLQL LERCVEDPET GLRLLPLKGA EKTEVVETTQ VYTEEETRRA FEETQIDIPG GGSHGGSSMS LWEVMQSNMI PEDQRARLMA DFQAGRVTKE RMIIIIIEII EKTEIIRQQN LASYDYVRRR LTAEDLYEAR IISLETYNLF REGTKNLREV LEMESAWRYL YGTGAVAGVY LPGSRQTLTI YQALKKGLLS AEVARLLLEA QAATGFLLDP VKGERLTVDE AVRKGLVGPE LHDRLLSAER AVTGYRDPYT EQTISLFQAM KKELIPAEEA LRLLDAQLAT GGIVDPRLGF HLPLEVAYQR GYLNKDTHDQ LSEPSEVRSY VDPSTDERLS YTQLLKRCRR DDPSGQMLLL LSDARKLTFR GLRKQITVEE LVRSQVMDEA TALQLQEGLT SIEEVTKNLQ KFLEGTSCIA GVFVDATKER LSVYQAMKKG IIRPGTAFEL LEAQAATGYV IDPIKGLKLT VEEAVRMGIV GPEFKDKLLS AERAVTGYKD PYSGKLISLF QAMKKGLILK DHGIRLLEAQ IATGGIIDPE ESHRLPVEVA YKRGLFDEEM NEILTDPSDD TKGFFDPNTE ENLTYLQLME RCITDPQTGL CLLPLKEKKR ERKTSSKSSV RKRRVVIVDP ETGKEMSVYE AYRKGLIDHQ TYLELSEQEC EWEEITISSS DGVVKSMIID RRSGRQYDID DAITKNLIDR SALDQYRAGT LSITEFADML SGNAGGFRSR SSSVGSSSSY PISSAGPRTQ LASWSDPTEE TGPVAGILDT ETLEKVSITE AMHRNLVDNI TGQRLLEAQA CTGGIIDPST GERFPVTEAV NKGLVDKIMV DRINLAQKAF CGFEDPRTKT KMSAAQALKK GWLYYEAGQR FLEVQYLTGG LIEPDTPGRV SLDEALQRGT VDARTAQKLR DVSAYSKYLT CPKTKLKISY KDALDRSMVE EGTGLRLLEA AAQSSKGYYS PYSVSGSGST AGSRTGSRTG SRAGSRRGSF DATGSGFSMT FSSSSYSSG YGRRYASGPS ASLGGPESAV A

Fig. (3). LC/MS/MS analysis revealed the binding of IQGAP1 and Plectin to A $\beta$. The stained bands were extracted and digested by trypsin. The samples were run on LC/MS/MS. Red color showed the detected amino acid sequence. A. myosin-9, B. actin, C. IQGAP1, D. Plectin. 


\section{Possible Biological and Pathological Meaning of the Binding of IQGAP1 and A $\beta$}

We have shown in this paper that $A \beta$ binds to IQGAP1, an effector of the Rho-family small GTPases Rac1 and Cdc42 $[11,12]$. A $\beta$ 1-42 originally penetrates cell membrane or ER membrane as a part of amyloid precursor protein (APP). A $\beta$ is produced by the sequential enzymatic actions of beta-site amyloid precursor protein-cleaving enzyme 1 (BACE-1), a $\beta$ secretase, and $\gamma$-secretase and exist inside cells and outside cells.

What the meaning of $A \beta$ and IQGAP1 binding in microglia? $A \beta$ could induce a local inflammatory reaction associated with regenerative changes in the surrounding neurons. Microglia produce multiple pro-inflammatory factors, including cytokines (tumor necrosis factor- $\alpha$ (TNF $\alpha$ ), interleukin (IL)-1, and IL-6), chemokines, reactive oxygen species $[8,13] \mathrm{A} \beta$ also produces $\mathrm{M}$ CSF and induces proliferation of microglia [8]. These results indicate that $A \beta$ induces microglial migration by chemokines and proliferation by M-CSF. The Rho-like GTPase, Rac1, induces cytoskeletal rearrangements required for cell migration and proliferation [14]. Vasiliev showed that an intact microtubule cytoskeleton was required to maintain the polarized distribution of actin-dependent protrusions at the leading edge of a migrating fibroblast [15]. RhoA mediates formation of contractile actin structures, such as stress fibres [16], and at the same time promotes stabilization of a sub-population of microtubules [17]. Rac kinase, which exists downstream of Rho kinase promotes contractility by increasing phosphorylation of the regulatory light chain of myosin-2 [18]. IQGAP1 binds to many proteins related to Rho kinase- microtubule cytoskeleton pathway including Racl and Cdc42 [19], myosin essential light chain [20]. Taken together, A $\beta$ binds to IQGAP1 and activates Rho kinase- microtubule cytoskeleton pathway to induce cellular migration and proliferation.

Plectin is the most versatile cytoskeletal linker protein known, which was first isolated nealy 30 years ago [21]. Plectin plays important roles in a number of cell functions including migration and wound healing [22]. Plectin has been shown to activate RhoA GTPase [23].

We have shown in this paper that $A \beta$ binds to several proteins inside cells. $A \beta$ may exist inside cells. Already it has been shown that monomeric synaptic $A \beta$, which stays at inside and outside cells may dampen excitatory transmission and prevent neuronal hyperactivity [23]. Early activation of signaling $[7,8]$ indicates that $\mathrm{A} \beta$ may work as a monomer or soluble oligomer, which may be inserted into cell membrane and endocytosed into cytoplasm. A $\beta$, which enter cells may bind to IQGAP1, Plectin and actin and work to migrate microglia by Rho-Rac1 signaling.

\section{ACKNOWLEDGEMENT}

We thank for manuscript preparation of N. Oiwa. This work was supported by the Ministry of Education, Science, Technology, Sports and Culture, Japan and the Global Centers of Excellence Program of Nagoya Unversity.

\section{CONFLICT OF INTEREST}

\author{
None declared.
}

\section{REFERENCES}

[1] Querfurth HW, LaFerla FM. Alzheimer's disease. N Engl J Med 2010; 362: 329-44.

[2] Akiyama H, Barger S, Barnum S, et al. Inflammation and Alzheimer's disease. Neurobiol Aging 2000; 21:383-421.

[3] Itagaki S, McGeer PL, Akiyama H, Zhu S, Selkoe D. Relationship of microglia and astrocytes to amyloid deposits of Alzheimer disease. J Neuroimmunol 1989; 24: 173-82.

[4] El Khoury JB, Moore KJ, Means TK, et al. CD36 mediates the innate host response to beta-amyloid. J Exp Med 2003; 197: 1657-66.

[5] Moore KJ, Freeman MW. Scavenger receptors in atherosclerosis: beyond lipid uptake. Arterioscler Thromb Vasc Biol 2006; 26: 1702-11.

[6] Stewart CR, Stuart LM, Wilkinson K, et al. CD36 ligands promote sterile inflammation through assembly of a Toll-like receptor 4 and 6 heterodimer. Nat Immunol 2010; 11: 155-61.

[7] Ito S, Sawada M, Haneda M, et al. Amyloid-beta peptides induce cell proliferation and macrophage colony-stimulating factor expression via the PI3-kinase/Akt pathway in cultured Ra2 microglial cells. FEBS Lett 2005; 579: 1995-2000.

[8] Ito S, Sawada M, Haneda M, Ishida Y, Isobe K. Amyloid-beta peptides induce several chemokine mRNA expressions in the primary microglia and $\mathrm{Ra} 2$ cell line via the PI3K/Akt and/or ERK pathway. Neurosci Res 2006; 56: 294-9.

[9] Walsh DM, Selkoe DJ. A beta oligomers - a decade of discovery. J Neurochem 2007; 101: 1172-84.

[10] Sawada M, Imai F, Suzuki H, Hayakawa M, Kanno T, Nagatsu T. Brainspecific gene expression by immortalized microglial cell-mediated gene transfer in the mammalian brain. FEBS Lett 1998; 433: 37-40.

[11] Fukata M, Kuroda S, Fujii K, et al. Regulation of cross-linking of actin filament by IQGAP1, a target for Cdc42. J Biol Chem 1997; 272: 2957983.

[12] Noritake J, Watanabe T, Sato K, Wang S, Kaibuchi K. IQGAP1: a key regulator of adhesion and migration. J Cell Sci 2005; 118: 2085-92.

[13] Heneka MT, O'Banion MK. Inflammatory processes in Alzheimer's disease. J Neuroimmunol 2007; 184: 69-91.

[14] Rodriguez OC, Schaefer AW, Mandato CA, Forscher P, Bement WM, Waterman-Storer CM. Conserved microtubule-actin interactions in cell movement and morphogenesis. Nat Cell Biol 2003; 5: 599-609.

[15] Vasiliev JM, Gelfand IM, Domnina LV, Ivanova OY, Komm SG, Olshevskaja LV. Effect of colcemid on the locomotory behaviour of fibroblasts. J Embryol Exp Morphol 1970; 24: 625-40.

[16] Etienne-Manneville S, Hall A. Rho GTPases in cell biology. Nature 2002; 420: 629-35.

[17] Cook TA, Nagasaki T, Gundersen GG. Rho guanosine triphosphatase mediates the selective stabilization of microtubules induced by lysophosphatidic acid. J Cell Biol 1998; 141: 175-85.

[18] Ridley AJ. Rho GTPases and cell migration. J Cell Sci 2001; 114: 2713-22.

[19] Kuroda S, Fukata M, Kobayashi K, et al. Identification of IQGAP as a putative target for the small GTPases, Cdc42 and Rac1. J Biol Chem 1996; 271: 23363-7.

[20] Weissbach L, Bernards A, Herion DW. Binding of myosin essential light chain to the cytoskeleton-associated protein IQGAP1. Biochem Biophys Res Commun 1998; 251: 269-76.

[21] Pytela R, Wiche G. High molecular weight polypeptides (270,000$340,000)$ from cultured cells are related to hog brain microtubuleassociated proteins but copurify with intermediate filaments. Proc Natl Acad Sci USA 1980; 77: 4808-12.

[22] Andrä K, Nikolic B, Stöcher M, Drenckhahn D, Wiche G. Not just scaffolding: plectin regulates actin dynamics in cultured cells. Genes Dev 1998; 12: 3442-51.

[23] Na S, Chowdhury F, Tay B, et al. Plectin contributes to mechanical properties of living cells. Am J Physiol Cell Physiol 2009; 296: C868-77. 